\title{
Full-Length Gonad Transcriptome Analysis of Amur Sturgeon Dmrt Family Genes: Identification, Characterization and Expression Patterns During Gonadal Differentiation
}

\author{
Xiujuan Zhang ( $\nabla$ zhangxj67@giz.gd.cn ) \\ Institute of zoology Guangdong Academy of Science \\ Guanyu Li \\ Instite of Zoology Guangdong Academy of Science \\ Jiabin Zhou \\ Instite of Zoology Guangdong Academy of Social Sciences \\ Mei Lv \\ Instite of Zoology Guangdong Academy of Sciences \\ Linmiao Li \\ Instite of Zoology Guangdong Academy of Sciences \\ Jinping Chen \\ Instite of Zoology Guangdong Academy of Sciences
}

\section{Research Article}

Keywords: Amur sturgeon, Acipenser schrenckii, Dmrt, sex differentiation, expression pattern

Posted Date: September 29th, 2021

DOI: https://doi.org/10.21203/rs.3.rs-926688/v1

License: (c) (i) This work is licensed under a Creative Commons Attribution 4.0 International License.

Read Full License 


\section{Abstract}

The regulatory mechanisms that govern sex differentiation in sturgeon are still poorly understood. The doublesex and Mab-3-related transcription factor (Dmrt) gene family is known for its extensive roles in sex determination and differentiation across vertebrates. This study aimed to identify new members of sturgeon Dmrt family genes and core actors in the gonadal differentiation of Amur sturgeon. A full-length gonad transcriptome database was exploited to identify Dmrt gene orthologs. Analyses of phylogenetic relationships and selection pressure were performed, and tissue expression profiles and spatiotemporal expression patterns in gonads were then analyzed using real-time PCR. In total, five Dmrt family genes were identified from the full-length gonad transcriptome, including Dmrt2, DmrtA1, DmrtA2, DmrtB1a and DmrtB1b. Phylogenetic analysis showed that these genes were clustered into clades corresponding to the doublesex/Mav-3 (DM) genes of vertebrates. Furthermore, the analysis of evolutionary selective pressure indicated that DmrtB1a and DmrtB1b were subject to positive selection, suggesting the existence of adaptive evolution in sturgeon. The extensive tissues expression profiling of each Dmrt family gene revealed typical characteristics. Remarkably, according to a spatiotemporal expression pattern analysis, DmrtA1 and DmrtA2 were predominantly distributed in undifferentiated gonads (UGs) and developing ovaries, whereas DmrtB1b showed the lowest expression in UGs. In later stages, DmrtB1b expression continuously increased in testes and was significantly higher in testes than in ovaries at 24 months after hatching $(\mathrm{M})$ and $36 \mathrm{M}$, which strongly suggests that DmrtB1b is a core regulatory factor involved in sexual differentiation in sturgeon. This study provides a genetic resource of full-length Dmrt family genes and increases the understanding of Dmrt functions in sex differentiation in sturgeon.

\section{Highlights}

1. Five Dmrt family genes with full-length transcript sequences were identified in Amur sturgeon.

2. The $\mathrm{Ka} / \mathrm{Ks}$ ratio values indicated that DmrtB1a and DmrtB1b were subject to positive evolutionary pressure.

3. Extensive tissue expression profiling of each Dmrt family gene showed typical characteristics.

4. DmrtB1b expression continuously increased and was significantly higher in developing testes.

\section{Introduction}

Sturgeons (Acipenseriformes) are an ancient fish group that originated during the Devonian period, with a history of over 200 million years; therefore, they constitute idea model species for studying development and evolution in vertebrates (Bemis et al., 1997; Saito et al., 2014). Sturgeon oocytes are highly prized as a value source of caviar food, referred to as "black gold"; however, wild sturgeon resources have declined in abundance due to habitat destruction and heavy exploitation. To date, in an updated January 2021 press release, the International Union for Conversation of Nature and Natural Resource (IUCN) Red List identified sturgeons as one of the most endangered animal groups, as some $85 \%$ of sturgeon species are on the verge of extinction (http://www.iucnredlist.org/search). Fortunately, efforts to develop a sturgeon 
aquaculture industry for artificial production have been made worldwide, which will not only ensure a stable supply of sturgeon products but will aid in the conservation of sturgeon stocks.

Although sturgeons are dioecious, it is difficult to distinguish females and males using morphological characteristics in larval, juvenile or even adult stages. The aquaculture water temperature does not affect the sex ratio (i.e., the sex differentiation of sturgeon shows no relationship with the breeding environment). More excellent female stocks or female gonadal development regulation technologies are urgently desired in the sturgeon aquaculture industry. Although the genomes of two sturgeon species have been decoded, and the American paddlefish (Polyodon spathula) genome has revealed chromosomal evolution mechanism (Cheng et al., 2021) while the Sterlet (Acipenser ruthenus) genome has provided insight into the mechanisms of segmental redipoidization (Du and Stöck, 2020), the regulatory mechanisms of sex differentiation in sturgeon are still poorly understood (Wei Wang et al., 2019).

The existence of doublesex and Mab-3-related transcription factor (Dmrt) genes was first formally reported in the fruit fly, Drosophila (Hildreth, 1965), in which a primary transcript common to the two sexes but produced male- and female- mRNAs (Burtis and Baker, 1989). Dmrt family genes are characterized by a doublesex/Mab-3 (DM) domain, which consists of six conserved intertwined CCHC and HCCC Zn (2+)- binding sites and a-helix structures (Erdman and Burtis, 1993; Zhu et al., 2000). In mouse, eight Dmrt genes (Dmrt1 to Dmrt8) have been identified (Veith et al., 2006a). Dmrt genes have been intensively studied because they represent major transcription factors of the sex determination and differentiation pathways. To date, Dmrt genes have been suggested to be sex determination genes in medaka fish, Oryzias latipes (Matsuda et al., 2007); African clawed frog, Xenopus laevis (Yoshimoto et al., 2008); chicken, Gallus gallus (Smith et al., 2009); and daphnia, Daphnia magna (Kato et al., 2011). In several other species, Dmrt genes have been suggested to play a core role in gonad development (i.e., downstream of the sex differentiation cascade) because changes in their expression levels induce sexual abnormalities rather than sex reversal. For example, the loss of Dmrt1 causes mouse spermatogonia to precociously exit the normal spermatogonial program and enter meiosis (Matson et al., 2010).

The Dmrt gene orthologs present vary among different species. For example, five, six, five, seven, five and eight Dmrt family genes have been identified in zebrafish (Danio rerio), Japanese pufferish (Takifugu rubripes), Atlantic cod (Gadus morhua), Largemouth bass (Micropterus salmoides), zebra finch (Taeniopygia guttata) and mouse (Mus musculus), respectively (Dong et al., 2020; Johnsen and Andersen, 2012; Veith et al., 2006a; Yamaguchi et al., 2006; Zhou et al., 2008). Dmrt1- 5 have been widely reported in all vertebrates, while Dmrt7 (DmrtC2) and Dmrt8 (DmrtC1) are only present in mammals. An earlier study implied that Dmrt6 (DmrtB1) may be missing in teleost fish (Veith et al., 2006b). Since a DmrtB1 ortholog was first reported to exist in a coelacanth (Latimeria chalumnae), studies have showed that Nile tilapia (Oreochromis niloticus) and five other teleost fish species, (i.e., largemouth bass (Micropterus salmoides), Asian seabass (Lates calcarifer), channel catfish (Ictalurus punctatus), spotted gar (Lepissosteus oculatus), and African coelacanth (Protopterus amphibius)) also carry DmrtB1 genes in their genome (Dong et al., 2020; Zhang et al., 2014b). Evolutionarily, it has been hypothesized that 
DmrtB1 probably separated from the ancestor of the Dmrt1-2 and Dmrt3-5 clades during the first round of vertebrate genome duplication (Johnsen and Andersen, 2012). Addtionally, synteny analysis in a genome-wide comparison indicated that all six other Dmrt family genes (Dmrt1, Dmrt2a, Dmrt2b and Dmrt3-5) are relatively conserved, but apart from DmrtB1 gene (Dong et al., 2020). Ancestral vertebrate genomes likely underwent two rounds (1R and $2 \mathrm{R}$ ) of whole-genome duplication (WGD). Teleost fishes then underwent a teleost-specific round of WGD (Dassau et al.). Furthermore, Acipenseriformes (sturgeons) experienced up to three additional lineage-specific WGDs (Lebeda et al., 2020; Ludwig et al., 2001), resulting in the polyploidy patterns observed in sturgeons. Polyploidy provides beneficial genetic flexibility and broad adaptive responses, i.e., additional gene copies theoretically allow evolution under reduced selective constraint and the acquisition of novel gene functions that contribute to adaptation (Panchy and Lehti-Shiu, 2016). The members of the Dmrt gene family in sturgeon are unclear. A previous study indicated that sexually dimorphic expression of Dmrt1 was not observed in the gonads of juvenile sturgeon (age $\leq 4$ years old) (Hatsumi Okada, 2017). Therefore, the core Dmrt genes involved in sex differentiation in sturgeon are still poorly understood because limited gene information is currently available.

The present study aimed to identify new members of the sturgeon Dmrt gene family and to reveal the core actors in gonadal differentiation in Amur sturgeon, Acipenser schrenckii. First, we identified five additional Dmrt family genes using a full-length gonad transcriptome sequencing database combined with molecular cloning. Then, the characteristics of these genes, including their sequence structure, phylogenetic relationship and selection pressure, were analyzed. Finally, we examined the expression distribution of five Dmrt family genes in seven main tissues, together with the spatiotemporal expression patterns in the gonads of $A$.schrenckii during sex differentiation and subsequent development. As a result, DmrtB1a and DmrtB1b are reported for the first time in sturgeon, and the spatiotemporal expression pattern of DmrtB1b may shed light on its core role in the gonads of Amur sturgeon during gonadal differentiation.

\section{Materials And Methods}

Identification of Dmrt family genes in Amur sturgeon

A full-length gonad transcriptome dataset was reported by the previous study (Zhang et al., 2020) and deposited in the NCBI database (accession number SRR9656303). Full-length Dmrt transcript candidates were further screened from NCBI nonredundant protein sequence (NR) database annotations using the Diamond BLASTX method according to E-value $<1 \times 10^{-5}$. After BLAST alignment and sequence homology assessment, five Dmrt genes with full-length sequences were identified and named Dmrt2, DmrtA1, DmrtA2, DmrtB1a and DmrtB1b. The detailed sequence information of the five Dmrt family genes is provided in Supplementary File 1.

Sequence analysis and structural characterization 
The open reading frames (ORFs) of five Dmrt genes were predicted using the NCBI ORFfinder program (www.ncbi.nlm.nih.gov/orffinder/) and the simple modular architecture research tool (SMART) was used to predict the conserved domains based on sequence homology (http://smart.embl-heidelberg.de/). Subsequently, the amino acids size, molecular weight (MW) and theoretical isoelectric point (PI) of each Dmrt protein were calculated by using ProtParam of the ExPASy database (http://web.expasy.org/protparam/).

The mature structure of each Dmrt protein was analyzed. The secondary structure was predicted using online PSIPRED software (https://bioinf.cs.ucl.ac.uk/psipred/). A tertiary protein structure model was constructed and further processed by using the SWISS-MODEL procedure (https://swissmodel.expasy.org/interactive/) and PyMO L annotation software. The subcellular localizations of five Dmrt proteins were revealed using the PedictPotein tool (https://www.predictprotein.org/).

Sequence alignment and phylogenetic analysis

Phylogenetic analysis was conducted with reference Dmrt proteins from eighteen species, including $A$. schrenckii, A. transmontanus, A. ruthenus, A. baerii, Parus major, Columba livia, Mus musculus, Homo sapiens, Bos Taurus, Takifugu rubripes, Paralichthys olivaceus, Oryzias latipes, Danio rerio, Oreochromis niloticus, Xenopus laevis, Nipponia Nippon, Anas platyrhynchos, and Gallus gallus. Dmrt accession numbers of eighteen typical species from eight classes are listed in Supplementary Table 1. The amino acid sequences of Dmrt genes were aligned using Clustal Omega (www.ebi.ac.uk/Tools/msa/clu-stalo/). A phylogenetic tree was reconstructed based on the maximum likelihood method using the MEGA $X$ package, and the number of bootstrap test repetitions was 1000 .

Furthermore, a phylogenetic tree of DmrtB1 amino acid sequences was reconstructed using mouse Dmrt8 as an outgroup. In addition to the DmrtB1a and DmrtB1b amino acid sequences of A.schrenckii employed for alignment, DmrtB1 sequences form five actinopterygian (ray-finned) fishes (Oreochromis niloticus, Micropterus salmoides, Lates calcarifer, Ictalurus punctatus, and Lepisosteus oculatus) and three sarcopterygian fishes (Protopterus amphibious, Latimeria chalumnae, and Latimeria menadoensi) were also included. The accession numbers of the DmrtB1 nucleotide and protein sequences from the eight species and mouse, as an outgroup are listed in Supplementary Table 2. The neighbor-joining method was used to reconstruct the phylogenetic tree by using the MEGA 7.0 package with the following parameter settings: Jones-Taylor-Thornton (JTT) model and all sites treatment. The values represent the bootstrap scores from 1000 trials, indicating the credibility of each branch.

Substitution rate estimation and comparison (Ka/Ks analysis)

The average nonsynonymous substitutions (Ka) and synonymous substitutions (Ks) of DmrtB1 and the corresponding $\mathrm{Ka} / \mathrm{Ks}$ ratios were calculated to test selection pressure at the codon-based nucleotide sequence level using KaKs_Calculator version 2.0 (Wang et al., 2010). The accession numbers of DmrtB1 genes with complete open reading frame (ORF) sequences from eight teleost fishes are listed in 
Supplementary Table 2. Thus, the Ka, Ks and $\mathrm{Ka} / \mathrm{Ks}$ values of all paired comparison groups based on the ORF sequences of DmrtB1 genes were determined. The $\mathrm{Ka} / \mathrm{Ks}$ ratio of each pair was calculated using three different algorithms, including the YN, gYN and MYN algorithms.

Expression assay of five Dmrt family genes using real-time PCR method

A tissue expression profiling assay was performed on seven main tissues of six 3 year-old Amur sturgeons, including the brain, intestine, muscle, liver, heart, spleen, and gill. Then, we further analyzed the spatiotemporal expression patterns in the gonads during their differentiation in four stages, ranging from undifferentiated gonads (UGs; Amur sturgeons at five months after hatching (M)) to differentiated gonads (testes and ovaries; $12 \mathrm{M}, 24 \mathrm{M}$ and $36 \mathrm{M}$ ).

Total RNA was extracted using TRIzol reagent and subsequently reverse transcribed using a First-strand cDNA Synthesis Kit (TaKaRa, Tokyo, Japan) according to the manufacturer's instructions. The reactions were incubated at $42^{\circ} \mathrm{C}$ for $60 \mathrm{~min}$ and $70^{\circ} \mathrm{C}$ for $15 \mathrm{~min}$ and then held at $4^{\circ} \mathrm{C}$. The primer sequences used in these assays are listed in Supplementary Table 3. Real-time PCR was performed using the Applied Biosystems Quant-Studio ${ }^{\text {TM }} 5$ platform (Thermo Fisher Scientific, Waltham, USA) according to the manufacturer's protocol. A total of $0.5 \mu \mathrm{l}$ of cDNA was used as the template in a $20 \mu \mathrm{l}$ reaction mixture, along with $10 \mu$ l of the SYBR® Green Master Mix (Applied Biosystems, Carlsbad, USA), $0.5 \mu$ l of each primer $(10 \mu \mathrm{M})$ and $8.5 \mu \mathrm{l}$ of ultrapure water under the following conditions: $50^{\circ} \mathrm{C}$ for $2 \mathrm{~min}$ for Heatedlabile Uracil-DNA Glycosylase (UDG) activation and then $95^{\circ} \mathrm{C}$ for $2 \mathrm{~min}$, followed by 40 cycles of $95^{\circ} \mathrm{C}$ for $15 \mathrm{~s}, 60^{\circ} \mathrm{C}$ for $30 \mathrm{~s}$ and $72^{\circ} \mathrm{C}$ for $30 \mathrm{~s}$.

Each targeted gene was analyzed in triplicate wells in more than three Amur sturgeon individuals (biological replicates). The expression levels of targeted genes were calculated using the relative quantity $\left(2^{-\triangle \triangle \mathrm{CT}}\right)$ method after normalization against the $\beta$-actin gene. Furthermore, the values obtained in intestines (as reference tissues for tissue expression profiling analysis) and UGs (as reference tissues for spatiotemporal expression pattern analysis) were normalized to 1 . The mean and standard error of the measurements $(\mathrm{M} \pm \mathrm{SEM})$ were calculated from the technological and biological replicates. Statistical significance was measured using the independent samples $t$-test in SPSS 17.0, with $P<0.05$ indicating significance.

\section{Results}

Sequence characteristics and conservation analysis of the five Dmrt family genes of $A$. schrenckii

The ORF of Dmrt2 has a total length of 1046 bp, encoding 348 amino acids. The ORFs of DmrtA1 and DmrtA2 are 1353 bp and 1338 bp and encode 450 and 445 amino acids, respectively. The ORFs of DmrtB1a and DmrtB1b are 1104 bp and 1122 bp and encode 367 and 373 amino acids, respectively. The detailed characteristics of the five Dmrt genes are summarized in Table 1. 
According to domain composition, the five Dmrt family genes could be divided into two groups: the first group contained DmrtA1 and DmrtA2, with both DM and DMA domains, while the second group contained Dmrt2, DmrtB1a and DmrtB1b, all of which had only the DM domain (Fig. 1). The schematic illustration of amino acid conservation among the five Dmrt family genes showed that that their DM domain sequences were highly conserved and the DMA domains of DmrtA1 and DmrtA2 were also highly conserved; however, the degree of variation among amino acid sequence of the DM and DMA domains and other regions was relatively high. A schematic illustration of the amino acid sequence conservation of the five genes in A.schrenckii is shown in Supplementary Fig. 1.

Advanced protein structure prediction for the five Dmrt family genes of $A$. schrenckii

The secondary structures of all five Dmrt family proteins consisted of 4-peptide a-helices (4.63-10.06\%), $\beta$-sheets (1.33-3.45\%) and coils (83.11-93.73\%). The secondary structure types and their corresponding proportions among the five Dmrt family proteins of $A$. schrenckii are shown in Fig. 2 and Supplementary Table 4.

The tertiary structure analysis of the five Dmrt family proteins showed that DmrtA1 and DmrtA2 existed as monomers and that the other three Dmrt proteins formed homotrimers. Through further analysis, we found that serine (Ser)-rich regions were only distributed in the a-helices of Dmrt2, DmrtA1 and DmrtA2, while redundant alanine (Ala) residues were mainly distributed in $\beta$-sheets or junctions between $\beta$-sheets and a-helices (Fig. 3).

Sequence similarity comparison and phylogenetic analysis

The similarity of the amino acid sequences among the DM domains and coding sequences (CDSs) of the five Dmrt family genes was analyzed. We found that the similarity among DM domains was higher than that among CDSs. For example, the amino acid sequence similarity was highest between the DM domains of DmrtB1a and DmrtB1b, at $98.2 \%$, followed by that between DmrtA1 and DmrtA2 (94.4\%) and Dmrt2 and DmrtA2 (88.9\%). In contrast, the degree of CDS similarity between Dmrt2 and DmrtB1a was the same as that between Dmrt2 and DmrtB1b, at 51.9\%. The results of the comparisons of amino acid similarity among the five Dmrt family genes (DM domains vs. CDS) are listed in Supplementary Table 5.

Subsequently, the phylogenetic tree of the five Dmrt family genes was reconstructed based on the amino acid sequence of the DM domains. The phylogenetic analysis revealed that the Amur sturgeon DmrtA2, DmrtA1 and Dmrt2 sequences aggregated into single branches and then clustered together with the corresponding sequences of other teleost fishes. Additionally, we found that DmrtA2 showed the earliest evolutionary divergence from the Dmrt family and that DmrtA1 presented the closest phylogenetic relationship with DmrtA2, followed by Dmrt2. Importantly, Amur sturgeon DmrtB1a and DmrtB1b, which were the only two novel Dmrt family genes in sturgeon aggregated into a single clade and then clustered together with the sequences of other vertebrates (Fig. 4). 
Furthermore, using the mouse Dmrt8 amino acid sequence as the outgroup, we reconstructed proteinbased phylogenetic tree of DmrtB1 genes reported from nine teleost fishes, including six actinopterygian fishes and three sarcopterygian fishes. As shown in the phylogenetic tree (Fig. 5A), it was clear that the DmrtB1 proteins from three Perciformes species (O. niloticus, M. samoides, and L. calcarifer) were clustered into one clade, while the DmrtB1 proteins from one Acipenseriformes species ( $A$. schrenckii), two Coelacanthiformes species (L. chalumnae, L. menadoensis), one Dipnoi species (P. amphibius), one Siluriformes species (I. punctatus) and one Lepidosteiformes species (L. oculatus) were clustered into another clade. Additionally, the DmrtB1 proteins from Acipenseriformes (A. schrenckii) and Siluriformes (I. punctatus) showed the closest phylogenetic relationship. Intriguingly however, the phylogenetic relationships of the DmrtB1 proteins between Acipenseriformes (A. schrenckii) and three sarcopterygian fishes were closer than those between Acipenseriformes (A. schrenckii) and three Perciformes species as they belonged to the actinopterygians.

\section{DmrtB1a and DmrtB1b were positive selection}

$\mathrm{Ka}$ and Ks assessments provide very important markers used in studies of evolutionary selection on genes. The $\mathrm{Ka} / \mathrm{Ks}$ ratio can be used to assess whether genes are under selection pressure, where $\mathrm{a} \mathrm{Ka} / \mathrm{Ks}$ $>1$ indicates probable positive selection, while $\mathrm{Ka} / \mathrm{Ks}$ values close to 1 represent neutral evolution or relaxed selection, and $\mathrm{Ka} / \mathrm{Ks}<1$ (especially less than 0.5 ) indicates purifying selection. First, the $\mathrm{Ka} / \mathrm{Ks}$ ratios of $A$. schrenckii were assessed against eight related teleost species: $O$. niloticus, $M$. salmoides, $L$. calcarifer, I. punctatus, L. oculatus, P. amphibious, L. chalumnae, and L. menadoensis. The results showed average Ka/Ks values $>1$ for DmrtB1a (1.49) and DmrtB1b (1.73), indicating positive selection (Fig. 5B). The relatively $\mathrm{Ka} / \mathrm{Ks}$ ratios indicating potential adaptive evolution may reflect specific selection pressure on $A$. schrenckii. Alternatively, they could be a sign of increased variability of these particular proteins within a broader group of species. The DmrtB1 gene Ka/Ks ratios were also calculated for the rest of the paired sequence comparisons among the eight fish species (average $\mathrm{Ka} / \mathrm{Ks}$ ratio -1.728 ). The $\mathrm{Ka} / \mathrm{Ks}$ cross test showed that positive DmrtB1 gene selection events were widespread in teleost fish.

Tissue expression profiles and spatiotemporal expression patterns in gonads

To reveal the expression characteristics of the five Dmrt family genes, expression analysis of seven main tissues of three year-old $A$. schrenckii individuals was first performed using real-time PCR. Tissue expression profiles of the five Dmrt family genes are shown in Fig. 6. Here, we observed two obvious expression characteristics. 1) Relative expression was lowest in the brain, but each Dmrt gene was expressed in all seven tissues, which may indicate that the five Dmrt genes have extensive biological functions. 2) Dmrt2, DmrtA1, DmrtA2 and DmrtB1a were the most highly expressed in gills, but the expression level of DmrtB1b was the highest in the liver.

Subsequently, spatiotemporal expression patterns in the gonads during sex differentiation and further development were analyzed using real-time PCR. The results of the spatiotemporal expression patterns analysis present in Fig. 7. The expression level of Dmrt2 was highest in $5 \mathrm{M}$ gonad (UGs) and there were no differences between the testis and ovary in any of the three examined differentiated stages. DmrtA1 
and DmrtA2 showed similar spatiotemporal expression patterns, with relatively higher expression levels in $5 \mathrm{M}$ and in the ovaries in the other three developmental stages. In the $36 \mathrm{M}$ gonads, the relative expression of DmrtA1 and DmrtA2 was significantly higher in ovaries than in testes $(P<0.05)$. In contrast, the expression levels of DmrtB1a gradually increased in the testes from $12 \mathrm{M}$ to $36 \mathrm{M}$, and the significantly different expression levels were observed between testes than ovaries at $36 \mathrm{M}(P<0.05)$. Most interestingly, the expression pattern of DmrtB1b was impressive during the gonadal differentiation of $A$. schrenckii. The expression of DmrtB1b was lowest in UGs. Thereafter, the expression level of DmrtB1b was first increased at $12 \mathrm{M}$, but then gradually decreased in the ovaries from $12 \mathrm{M}$ to $36 \mathrm{M}$, while it continuously increased in testes and the testes showed significantly higher expression than the ovaries at $24 \mathrm{M}$ and $36 \mathrm{M}(P<0.05)$.

\section{Discussion}

The Dmrt gene family produces a large number of transcripts, which show highly conserved biological functions during sex determination and differentiation across vertebrates. One aim of the present study was to identify novel Dmrt gene family members in sturgeon. Dmrt genes can be divided into several different subfamilies according to protein domain, such as DmrtA, DmrtB, and DmrtC. In the DmrtA subfamilies, three members, DmrtA1 (Dmrt4) DmrtA2 (Dmrt5) and DmrtA3 (Dmrt3), also show conserved DMA domains, in addition to the DM domain (Ottolenghi et al., 2002). In comparison with short-read sequencing, the methodological advantages of PacBio Isoform sequencing (Iso-Seq) achieves better completeness of both the $5^{\prime}$ and $3^{\prime}$ ends sequences of full-length cDNA molecules. The further annotation of full-length transcripts can play a critical role in gene discovery (Zhang et al., 2019). Therefore, five additional full-length Dmrt family genes were successfully identified in A. schrenckill, which were designated Dmrt2, DmrtA1, DmrtA2, DmrtB1a and DmrtB1b. These findings provide a genetic basis for better understanding the structural and functional properties of these genes in sturgeon species.

The Dmrt gene family has undergone frequent, independent gene replication events during the course of evolution; thus the kinds and members of Dmrt family genes may be different between phyla. Variation is even observed within vertebrates, among which mammals include some genes that are absent from teleost fish (Volff et al., 2003); for example, no fish orthologs of Dmrt7 and Dmrt8 have been identified (Zafar et al., 2019). In the present study, five Dmrt family genes with full-length cDNA sequences were identified as new family members from sturgeon, in addition to Dmrt $1 \mathrm{a}$ and Dmrt $1 \mathrm{~b}$ reported previously (Hatsumi Okada, 2017). According to the phylogenetic relationship analysis (Fig. 4), the five Dmrt family genes belong to four different Dmrt subfamilies. We also found that the phylogenetic positions of Dmrt2, DmrtA1 and DmrtA2 were relatively ancient, which was consistent with a previous study (Volff et al., 2003).

Some Dmrt family genes in different species may show novel expression patterns and new biological functions. For example, Dmrt1 gave rise to the master male-determinating gene found in medaka fish (Matsuda et al., 2007) but not in more divergent fish. In zebrafish, the duplicated Dmrt2b and Dmrt2a with structural variation and expression diversification suggests their diverse roles, as Dmrt2b functions in 
specification of branchial arches, and Dmrt2a functions in somitogenesis (Zhou et al., 2008). In the present study, extensive tissues expression profiling revealed interesting and common expression characteristics of the five Dmrt family genes. Among these genes, Dmrt2, DmrtA1, DmrtA2 and DmrtB1a were highly expressed in gills, but DmrtB1b showed the most abundant expression in the liver. Contrasting expression patterns of these genes in tissues other than the gonads were initially reported; thus their functions may be worthy of further investigation.

The roles of Dmrt family genes in sex determination and differentiation vary among vertebrates. For example, Dmrt1 has been identified as a sex determination gene in 0 . latipes (Matsuda et al., 2007), $X$. laevis (Yoshimoto et al., 2008), and G. gallus (Smith et al., 2009), but no role of this gene has been identified in sturgeon (Hatsumi Okada, 2017). In the present study, five additional Dmrt family genes with full-length cDNA sequences were identified for the first time, which represents the newest genetic information on Dmrt family genes in sturgeon. Further analysis revealed the spatiotemporal expression patterns of the five Dmrt family genes in gonads in stages ranging from UGs to the ovaries or testes of three-year-old individuals. Our results showed that the expression of Dmrt2 was highest in UGs, whereas it appeared to play no role in early gonadal development in A. schrenckii. However, the role of Dmrt2 varies in other teleost fish. For example, the expression of Dmrt2 is higher in male gonads during early gonadal development in Chinese tongue sole (Cynoglossus semilaevis) (Zhu et al., 2019), and Dmrt2 has been shown to be involved in sex differentiation in swamp eel (Sheng et al., 2014). However, Dmrt2 is also reported to play a significant role in premature ovarian differentiation and maintenance in orange-spotted grouper (Epinephelus coioides) (Lyu et al., 2019). DmrtA1 was demonstrated to be absent in the zebrafish genome, but DmrtA2 was found to be preferentially expressed in the adult testis (Xu et al., 2013). In the present study, it was interesting to find that DmrtA1 and DmrtA2 were highly expressed in UGs and in the ovaries in the other three developmental stages, with significantly higher expression being observed in ovaries than in testes in $36 \mathrm{M} \mathrm{A}$. schrenckii. Our results are consistent with the role of DmrtA2 in normal female germ cell development in humans (Poulain et al., 2014).

Dmrt1 shows testis-dominant expression in adults (i.e., four-year-old $A$. schrenckii) but not in the gonads of juvenile individuals (Hatsumi Okada, 2017). However, DmrtB1b presented the lowest expression in UGs in the present study, after which it continuously increased in testes and presented significantly higher expression in testes than in ovaries in $24 \mathrm{M}$ and $36 \mathrm{M} \mathrm{A}$. schrenckii. According to related studies, $24 \mathrm{M}$ and $36 \mathrm{M}$ correspond to the stages of spermatogonial cell proliferation and early gametogenesis, respectively, in $A$. schrenckii (Zhang et al., 2020). The above observation may suggest that DmrtB1b acts as a core regulatory factor in the sexual differentiation of $A$. schrenckii, DmrtB1a and DmrtB1b were reported in sturgeon for the first time in the present study. A functional analysis of DmrtB1 ${ }^{-/-}$mice showed that DmrtB1 played a key role in coordinating the transition in gametogenic programs from spermatogonial differentiation and mitosis to spermatocyte development and meiosis (Zhang et al., 2014a). The mammalian results may suggest core roles of DmrtB1b in male gonad differentiation in sturgeon, which is worth further study. 
In conclusion, a total of five new members of the Dmrt gene family were identified in sturgeon based on a full-length gonad transcriptome database; these genes were designated Dmrt2, DmrtA1, DmrtA2, DmrtB1a and DmrtB1b. These five Dmrt proteins all contain a conserved DM domain and/or DMA domain and exhibit similar mature structures. Phylogenetic analysis indicated that these genes were clustered into clades corresponding other DM domain genes of vertebrates. Some interesting characteristics of DmrtB1a and DmrtB1b were also found; for example, they showed closer phylogenetic relationships between Acipenseriformes (A.schrenckii) and three sarcopterygian fish groups and positive selection pressure in sturgeon. Extensive tissue expression profiling of each Dmrt family gene showed typical characteristics. Remarkably, the spatiotemporal expression pattern of DmrtB1b may shed light on its core role in the gonads of Amur sturgeon during sex differentiation and further testis development. Overall, this study not only provides a genetic resource of full-length Dmrt family genes but also increases available knowledge for the further investigation of Dmrt functions in sex differentiation in sturgeon.

\section{Declarations}

\section{Author Declarations}

\section{Funding}

This work was supported by the National Natural Science Fund of China [31802279], the GDAS' Project of Science and Technology Development [2020GDASYL-20200104026], and the Key Laboratory Fund of Animal Protection and Resource Utilization of Guangdong Province [GIABR-KF201704].

\section{Conflicts of interest}

The authors declare that they have no competing interests.

\section{Ethics approval}

All experimental animal procedures followed the principles of Guide for Care and Use of Laboratory Animal and were approved by the Ethics Committee of Laboratory Animal of Institute of Zoology, Guangdong Academy of Science (NO: GIZ20200828).

\section{Consent to participate}

Consent was obtained from individual participants.

\section{Consent to publication}

All authors have signed an agreement to publish this article.

\section{Availability of data and material}


All data generated or analyzed during this study are included in this published article and its supplementary files.

\section{Code availability}

Not applicable.

\section{Authors' contributions}

XJ Zhang and JP Chen conceived and designed the study. XJ Zhang, GY Li, JB Zhou, M Lv and LM Li performed the experiments. XJ Zhang and GY Li analyzed the sequence data. XJ Zhang contributed to the writing of the manuscript. All authors read and approved the final manuscript.

\section{References}

1. Bemis WE, Findeis EK, Grande L (1997) An overview of Acipenseriformes. In Sturgeon biodiversity and conservation (Springer), pp. 25-71

2. Burtis KC, Baker BS (1989) Drosophila doublesex gene controls somatic sexual differentiation by producing alternatively spliced mRNAs encoding related sex-specific polypeptides. Cell 56:997-1010

3. Cheng P, Huang Y, Lv Y, Du H, Ruan Z, Li C, Ye H, Zhang H, Wu J, Wang C, Ruan R, Li Y, Bian C, You X, Shi C, Han K, Xu J, Shi Q, Wei Q (2021) The American paddlefish genome provides novel insights into chromosomal evolution and bone mineralization in early vertebrates. Mol Biol Evol 38:1595-1607

4. Dassau E, Zisser H, Palerm C, Buckingham CA, Jovanovic B, Doyle LJ F (2008) Modular artificial beta-cell system: a prototype for clinical research. J Diabetes Sci Technol 2:863-872

5. Dong J, Li J, Hu J, Sun C, Tian Y, Li W, Yan N, Sun C, Sheng X, Yang S, Shi Q, Ye X (2020) Comparative genomics studies on the dmrt gene family in fish. Frontiers in genetics 11:563947

6. Du K, Stöck M (2020) The sterlet sturgeon genome sequence and the mechanisms of segmental rediploidization. Nat Ecol Evol 4(6):841-852

7. Erdman SE, Burtis KC (1993) The Drosophila doublesex proteins share a novel zinc finger related DNA binding domain. Embo J 12:527-535

8. Okada H, Hagihara S, Yamashita K, ljiri S, Adachi S (2017) Expression pattern of foxl2 and dmrt1 in gonad of Amur sturgeon Acipenser schrenckii in relation to sex differentiation. Aquaculture 479:712720

9. Hildreth PE (1965) Doublesex, recessive gene that transforms both males and females of Drosophilainto intersexes. Genetics 51:659-678

10. Johnsen $H$, Andersen $\varnothing$ (2012) Sex dimorphic expression of five dmrt genes identified in the Atlantic cod genome. The fish-specific dmrt2b diverged from dmrt2a before the fish whole-genome duplication. Gene 505:221-232

11. Kato $Y$, Kobayashi $K$, Watanabe H, Iguchi $T$ (2011) Environmental sex determination in the branchiopod crustacean Daphnia magna: deep conservation of a Doublesex gene in the sex- 
determining pathway. PLoS Genet 7:e1001345

12. Lebeda I, Ráb P, Majtánová Z, Flajšhans M (2020) Artificial whole genome duplication in paleopolyploid sturgeons yields highest documented chromosome number in vertebrates. Scientific reports 10:19705

13. Ludwig A, Belfiore NM, Pitra C, Svirsky V, Jenneckens I (2001) Genome duplication events and functional reduction of ploidy levels in sturgeon (Acipenser, Huso and Scaphirhynchus). Genetics 158:1203-1215

14. Lyu Q, Hu J, Yang X, Liu X, Chen Y, Xiao L, Liu Y, Wang Q, Chen J, Huang M, Yu HR, Shi HR, Zhang Y, Zhao HH (2019) Expression profiles of dmrts and foxls during gonadal development and sex reversal induced by 17a-methyltestosterone in the orange-spotted grouper. Gen Comp Endocrinol 274:26-36

15. Matson CK, Murphy MW, Griswold MD, Yoshida S, Bardwell VJ, Zarkower D (2010) The mammalian doublesex homolog DMRT1 is a transcriptional gatekeeper that controls the mitosis versus meiosis decision in male germ cells. Dev Cell 19:612-624

16. Matsuda M, Shinomiya A, Kinoshita M, Suzuki A, Kobayashi T, Paul-Prasanth B, Lau EL, Hamaguchi S, Sakaizumi M, Nagahama Y (2007) DMY gene induces male development in genetically female (XX) medaka fish. Proc Natl Acad Sci USA 104:3865-3870

17. Ottolenghi C, Fellous M, Barbieri M, McElreavey K (2002) Novel paralogy relations among human chromosomes support a link between the phylogeny of doublesex-related genes and the evolution of sex determination. Genomics 79:333-343

18. Panchy N, Lehti-Shiu M (2016) Evolution of gene duplication in plants. Plant Physiol 171(4):22942316

19. Poulain M, Frydman N, Tourpin S, Muczynski V, Souquet B, Benachi A, Habert R, Rouiller-Fabre V, Livera G (2014) Involvement of doublesex and mab-3-related transcription factors in human female germ cell development demonstrated by xenograft and interference RNA strategies. Molecular human reproduction 20:960-971

20. Saito T, Psenicka M, Goto R, Adachi S, Inoue K, Arai K, Yamaha E (2014) The origin and migration of primordial germ cells in sturgeons. PloS one 9:e86861

21. Sheng Y, Chen B, Zhang L, Luo M, Cheng H, Zhou R (2014) Identification of Dmrt genes and their upregulation during gonad transformation in the swamp eel (Monopterus albus). Molecular biology reports 41:1237-1245

22. Smith CA, Roeszler KN, Ohnesorg T, Cummins DM, Farlie PG, Doran TJ, Sinclair AH (2009) The avian Z-linked gene DMRT1 is required for male sex determination in the chicken. Nature 461:267-271

23. Veith AM, Klattig J, Dettai A, Schmidt C, Englert C, Volff JN (2006a) Male-biased expression of Xchromosomal DM domain-less Dmrt8 genes in the mouse. Genomics 88:185-195

24. Veith AM, Schäfer M, Klüver N, Schmidt C, Schultheis C, Schartl M, Winkler C, Volff JN (2006b) Tissue-specific expression of dmrt genes in embryos and adults of the platyfish Xiphophorus maculatus. Zebrafish 3:325-337 
25. Volff JN, Zarkower D, Bardwell VJ, Schartl M (2003) Evolutionary dynamics of the DM domain gene family in metazoans. Journal of molecular evolution 57(Suppl 1):S241-S249

26. Wang D, Zhang Y, Zhang Z, Zhu J, Yu J (2010) KaKs_Calculator 2.0: a toolkit incorporating gammaseries methods and sliding window strategies. Genomics Proteomics Bioinformatics 8:77-80

27. Wang W, Zhu H, Dong Y, Dong T, Tian ZH, Hu H (2019) Identification and dimorphic expression of sex-related genes during gonadal differentiation in sterlet Acipenser ruthenus, a primitive fish species. Aquaculture 500:178-187

28. Xu S, Xia W, Zohar Y, Gui JF (2013) Zebrafish dmrta2 regulates the expression of cdkn2c in spermatogenesis in the adult testis. Biol Reprod 88:14

29. Yamaguchi A, Lee KH, Fujimoto H, Kadomura K, Yasumoto S, Matsuyama M (2006) Expression of the DMRT gene and its roles in early gonadal development of the Japanese pufferfish Takifugu rubripes. Comparative biochemistry and physiology. Part D. Genomics proteomics 1:59-68

30. Yoshimoto S, Okada E, Umemoto H, Tamura K, Uno Y, Nishida-Umehara C, Matsuda Y, Takamatsu N, Shiba T, Ito M (2008) A W-linked DM-domain gene, DM-W, participates in primary ovary development in Xenopus laevis. Proc Natl Acad Sci U S A 105:2469-2474

31. Zafar I, Rather MA, Dhandare BC (2019) Genome-Wide identification of doublesex and Mab-3-Related transcription factor (DMRT) genes in nile tilapia (oreochromis niloticus). Biotechnology reports 24:e00398

32. Zhang T, Murphy MW, Gearhart MD, Bardwell VJ, Zarkower D (2014a) The mammalian Doublesex homolog DMRT 6 coordinates the transition between mitotic and meiotic developmental programs during spermatogenesis. Development 141:3662-3671

33. Zhang X, Li G, Jiang H, Li L, Ma J, Li H, Chen J (2019) Full-length transcriptome analysis of Litopenaeus vannamei reveals transcript variants involved in the innate immune system. Fish Shellfish Immunol 87:346-359

34. Zhang X, Wang H, Li M, Cheng Y, Jiang D, Sun L, Tao W, Zhou L, Wang Z, Wang D (2014b) Isolation of doublesex- and mab-3-related transcription factor 6 and its involvement in spermatogenesis in tilapia. Biol Reprod 91:136

35. Zhang X, Zhou J, Li L, Huang W, Ahmad HI, Li H, Jiang H, Chen J (2020) Full-length transcriptome sequencing and comparative transcriptomic analysis to uncover genes involved in early gametogenesis in the gonads of Amur sturgeon (Acipenser schrenckii). Frontiers in zoology 17:11

36. Zhou X, Li Q, Lu H, Chen H, Guo Y, Cheng H, Zhou R (2008) Fish specific duplication of Dmrt2: characterization of zebrafish Dmrt2b. Biochimie 90:878-887

37. Zhu L, Wilken J, Phillips NB, Narendra U, Chan G, Stratton SM, Kent SB, Weiss MA (2000) Sexual dimorphism in diverse metazoans is regulated by a novel class of intertwined zinc fingers. Genes Dev 14:1750-1764

38. Zhu Y, Cui Z, Yang Y, Xu W, Shao C, Fu X, Li Y, Chen S (2019) Expression analysis and characterization of dmrt2 in Chinese tongue sole (Cynoglossus semilaevis). Theriogenology 138:1-8 


\section{Tables}

Table 1. Sequence characteristics of the five Dmrt family genes and the encoded proteins in A. schrenckii.

\begin{tabular}{llllllll} 
Gene & $\begin{array}{l}\text { Full-length } \\
(\mathrm{bp})\end{array}$ & $\begin{array}{l}\text { ORF } \\
(\mathrm{bp})\end{array}$ & $\begin{array}{l}5^{\prime} \mathrm{UTR} \\
(\mathrm{bp})\end{array}$ & $\begin{array}{l}\text { 3'UTR } \\
(\mathrm{bp})\end{array}$ & $\begin{array}{l}\text { Amino } \\
\text { acids } \\
(\mathrm{aa})\end{array}$ & $\begin{array}{l}\text { molecular } \\
\text { weight } \\
(\mathrm{kDa})\end{array}$ & $\begin{array}{l}\text { Isoelectric point } \\
(\mathrm{PI})\end{array}$ \\
\hline Dmrt 2 & 2291 & 1046 & 324 & 921 & 348 & 38.7 & 8.57 \\
\hline $\begin{array}{l}\text { Dmrt } \\
\text { A1 }\end{array}$ & 1888 & 1353 & 381 & 769 & 450 & 48.9 & 8.70 \\
$\begin{array}{l}\text { Dmrt } \\
\text { A2 }\end{array}$ & 2853 & 1338 & 480 & 1035 & 445 & 47.8 & 8.35 \\
$\begin{array}{l}\text { Dmrt } \\
\text { B1a }\end{array}$ & 2434 & 1104 & 10 & 1113 & 367 & 39.5 & 7.19 \\
$\begin{array}{l}\text { Dmrt } \\
\text { B1b }\end{array}$ & 2348 & 1122 & 72 & 1155 & 373 & 40.9 & 9.80
\end{tabular}

Figures

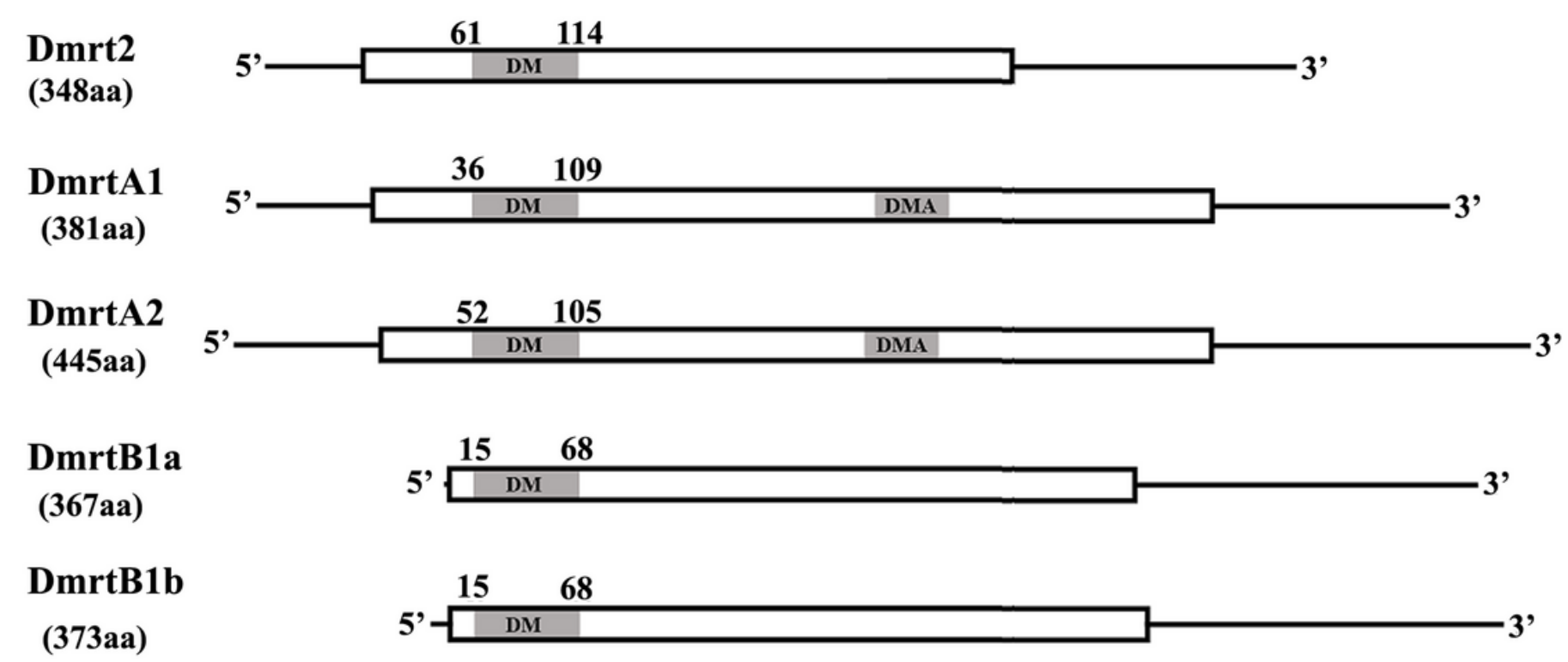

Figure 1

Schematic illustration of the primary structures of the five Dmrt family genes in A. schrenckii. 


\section{Dmrt2}

(348aa)

\section{DmrtA1}

(450aa)

\section{DmrtA2}

(445aa)

\section{DmrtB1a}

(367aa)

\section{DmrtB1b}

(373aa)
1 M T D L L V P

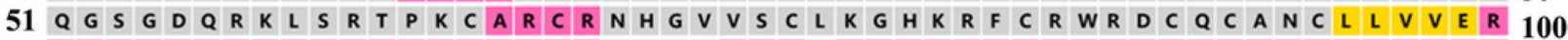

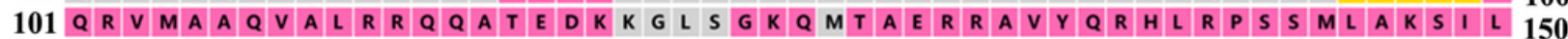

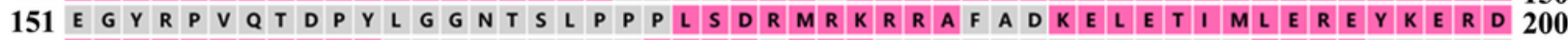

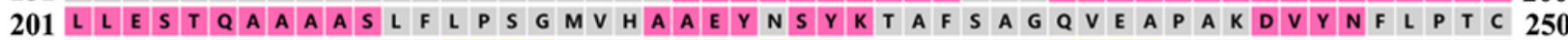

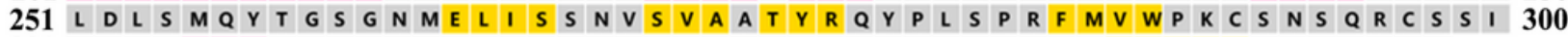

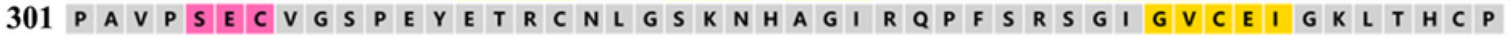

1 M D A T S S R P L L P S G L P G H S P L S L S V S G L Q M S S S L L R P P P L F L R R A A A C N P P S L 50

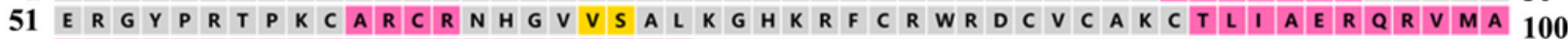

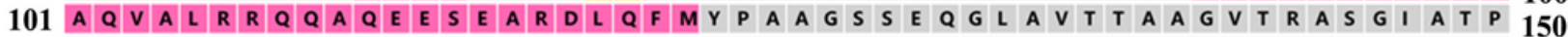
151 I T P C Y E V F G M G D Q K G E E K T H K Y D F Y S G F M G H P L L F P H T G Q M P S T L D K T S S 200

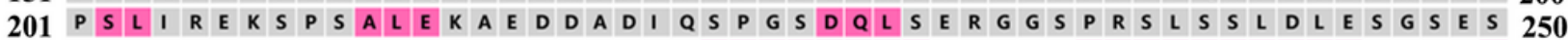
251 E $R$ R P K 301 V $Q$ Q A I I E $Q$ Q V L $L$ N S K E H K D N P N G T E S T V S E P G N L Q R S S S S F G L P G V V G L G L S M G L G 350

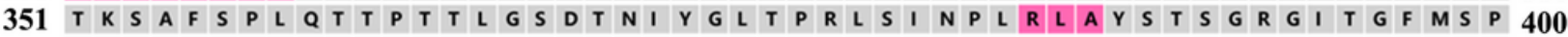
401 Y V T P G L M P A L Q F R P P M D Y S F P G M I R D F S Y F Q N K E S L C N T G L Y S R V N Q E N Q 450

1 ME L R A E I P T S Q P S S H V H P G A G D G A A N S I P V S S MA S S T F L R G P P L I L L R T T E K Y

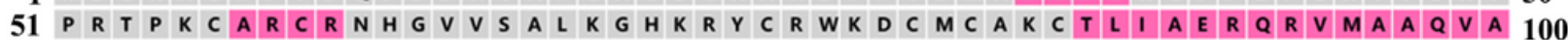

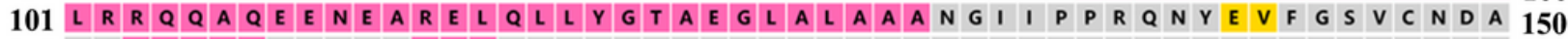
151 N S E E S K L 201 R H G S G S E NG DGE S F I S S P V S K A V K D GE D T P G S I S P L G S D S G S E T D K DE Q E 250

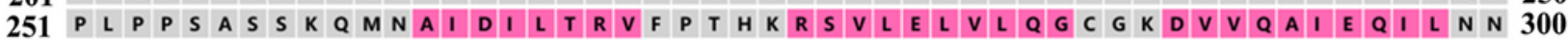

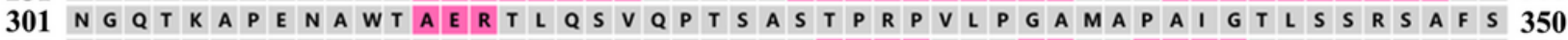
351 P L Q P P N G P H F G A D P T A Y P L S T H L G L N P L L R L A 401 P T L G F R P P M D Y A F S D L I R D R T N L H K D Q G Y T N G L Y G P M V N S N P D K P

1 M P H R P N P D I A E K R L R T P K C A R C R N H G F T V Q L L K G H S G K C A F L H C Q C W K C A L 50

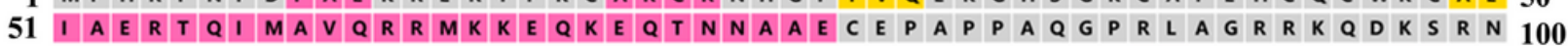
101 I D E $D$ C C C E A A E P A G A T $S$ A A R L L 151 G T S R R Q 201 S P G M D F H T G A D W P E R D G A A W S S G G WA A G G M Q G N A F R M A P R Y V P D G E L L $S$ S 251 V H R R P V P V K L Y S R C P C H T I F L N V D A G Q P C F H E P P L P V G G F H H F P G H S V Q Y S L 300 301 D P A Q G S G R A S Y Y A P P S V R P C L V Q D A S S Y S L L H P D S V V S P R E E E E E A S G G L C G S Q D D 350 351 S D V I S V G NE A S P QE NSG

1 M P H R P N P D I A E K R L R T P K C A R C R N H G F T V Q L K G H S G K C A F M H C Q C WKCAL 50

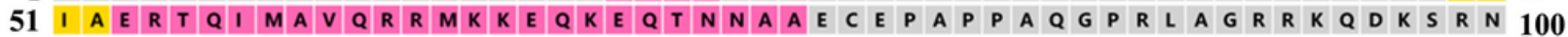
101 I D E D C R E A E P A G A T S A R L L N 151 G T S S R Q F D D A G R M H T A F G V S A P V Q T A A P E T A L 201 S P G M D F H T G A D W P E R D G A A W S S G G WA A G G M Q G N A F R M A P R Y V P D G E L L S A 250 251 V $H$ H R P $P$ V P P V K L L Y S S R Y P P C H A I F

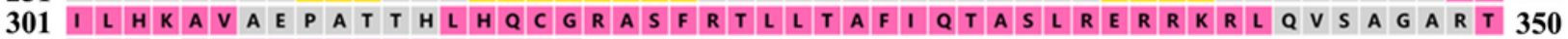
\begin{tabular}{ll|l|l|l|l|l|l|l|l|l|l|l|l|l|l|l|l|l|l|l}
351 & L & M S & F & P & W & G & L & R & R & L & L & $R$ & K & T & A & V & N N N R & I & I & H
\end{tabular}
Helix

\section{Strand}

Figure 2

Schematic illustration of the secondary structures of the five Dmrt family proteins in A. schrenckii. 


\section{Dmrt2}

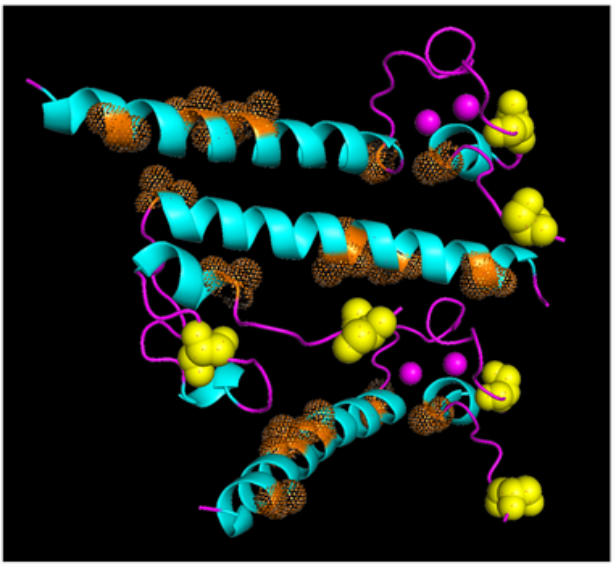

\section{DmrtB1a}

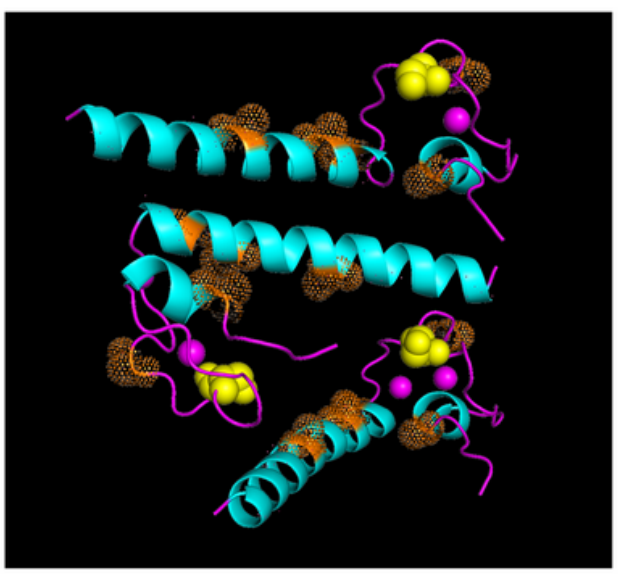

DmrtA1

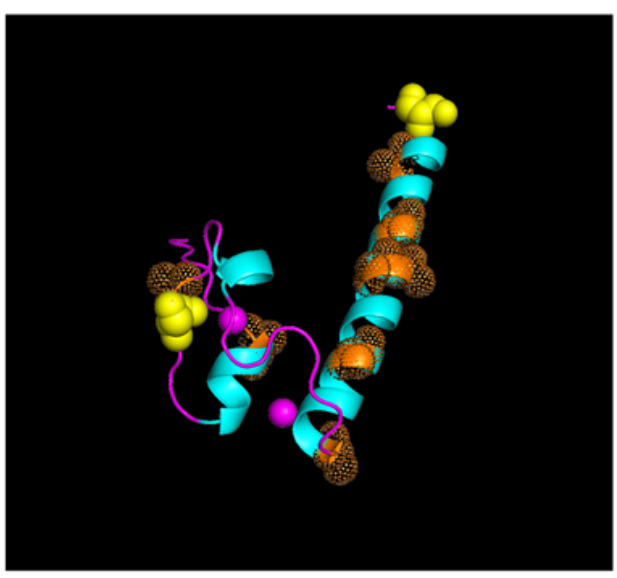

\section{DmrtB1b}

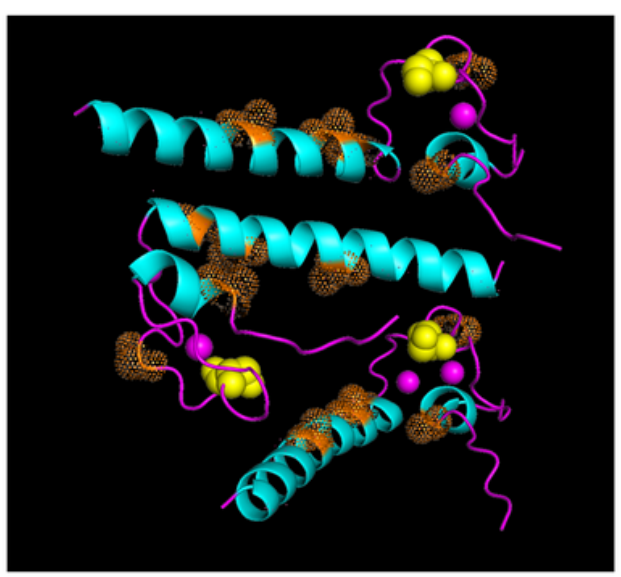

DmrtA2
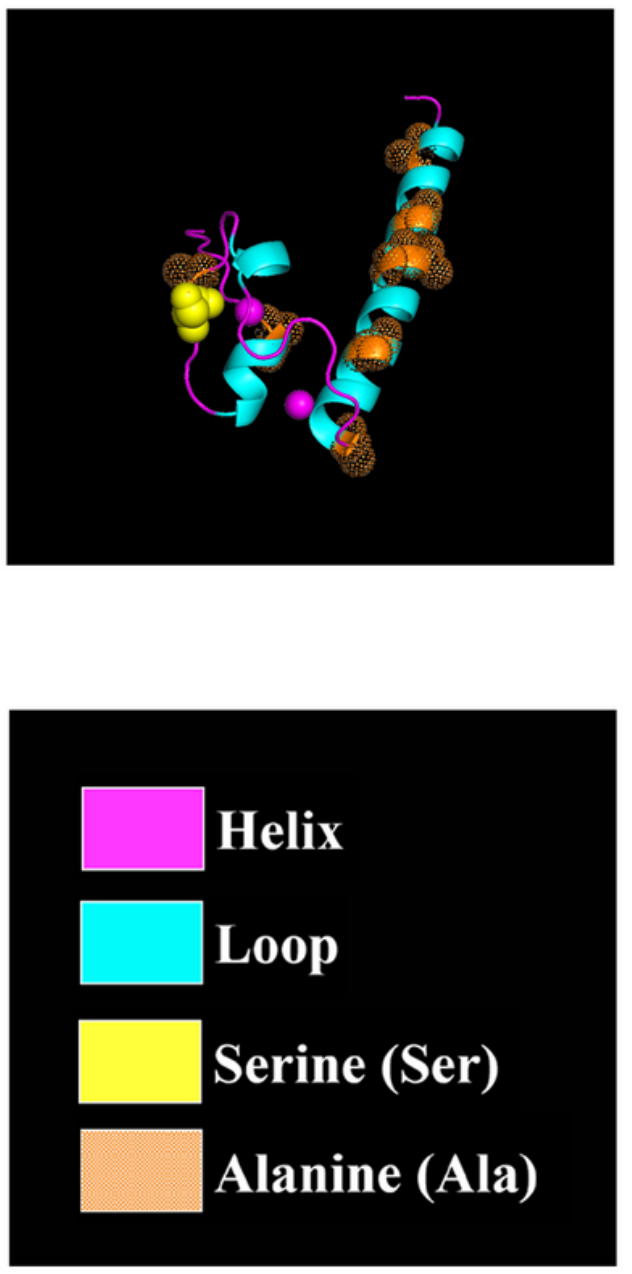

Figure 3

Schematic illustration of the tertiary structures of the five Dmrt family proteins in A. schrenckii. 


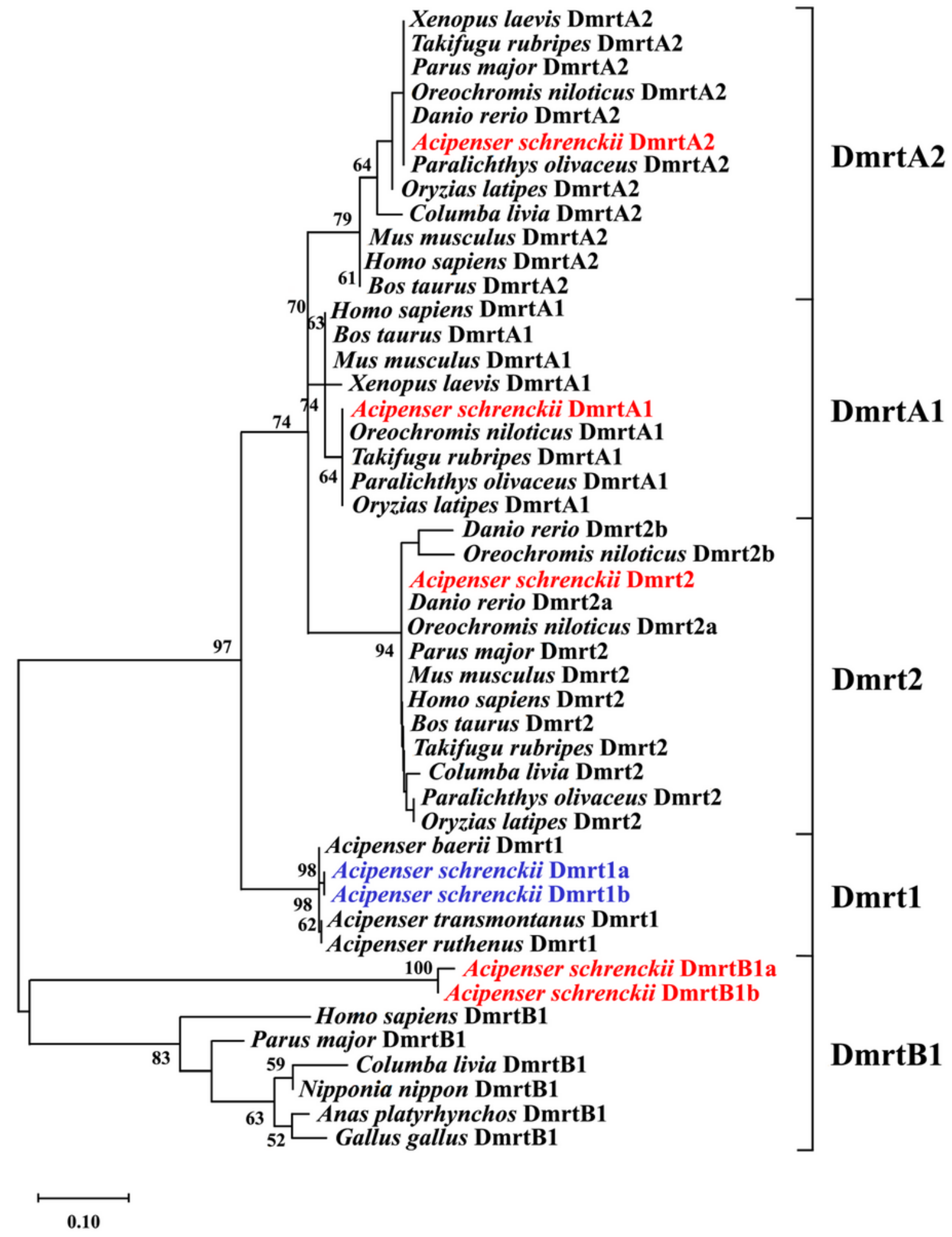

Figure 4

Maximum likelihood analysis showing phylogenetic relationships based on the amino acid sequences of the Dmrt gene DM domains among 19 species. The numbers on each branch represent the bootstrap values obtained with 1000 replicates. 
A

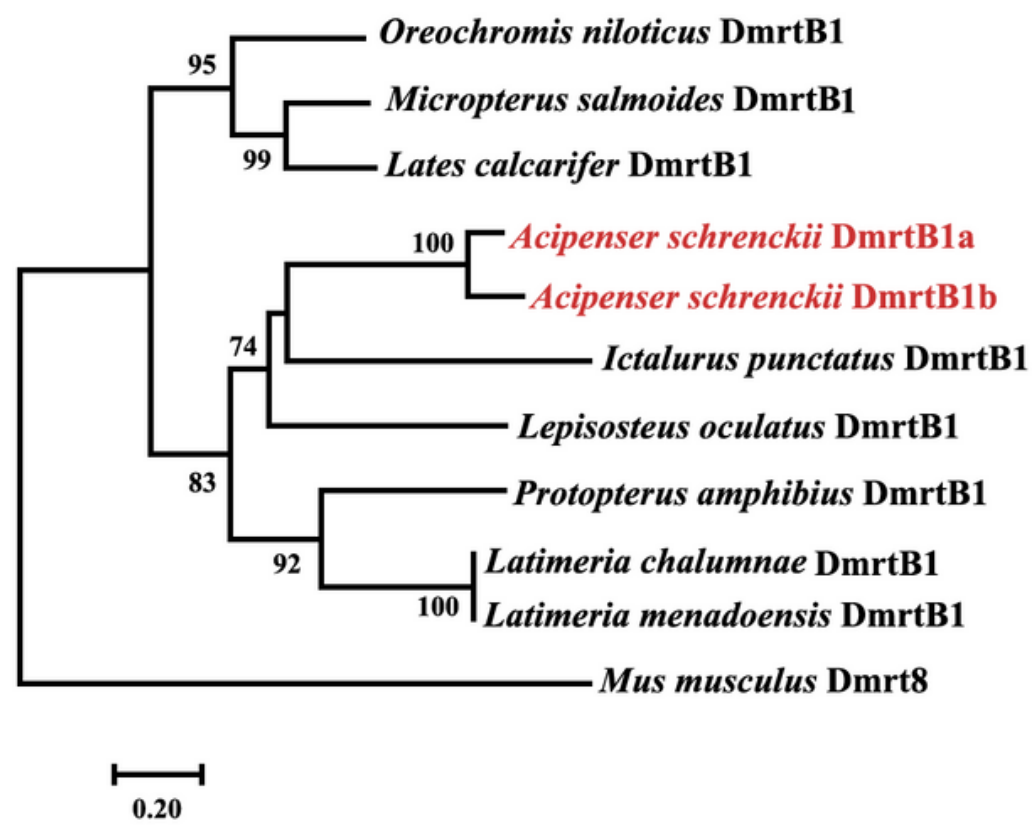

B

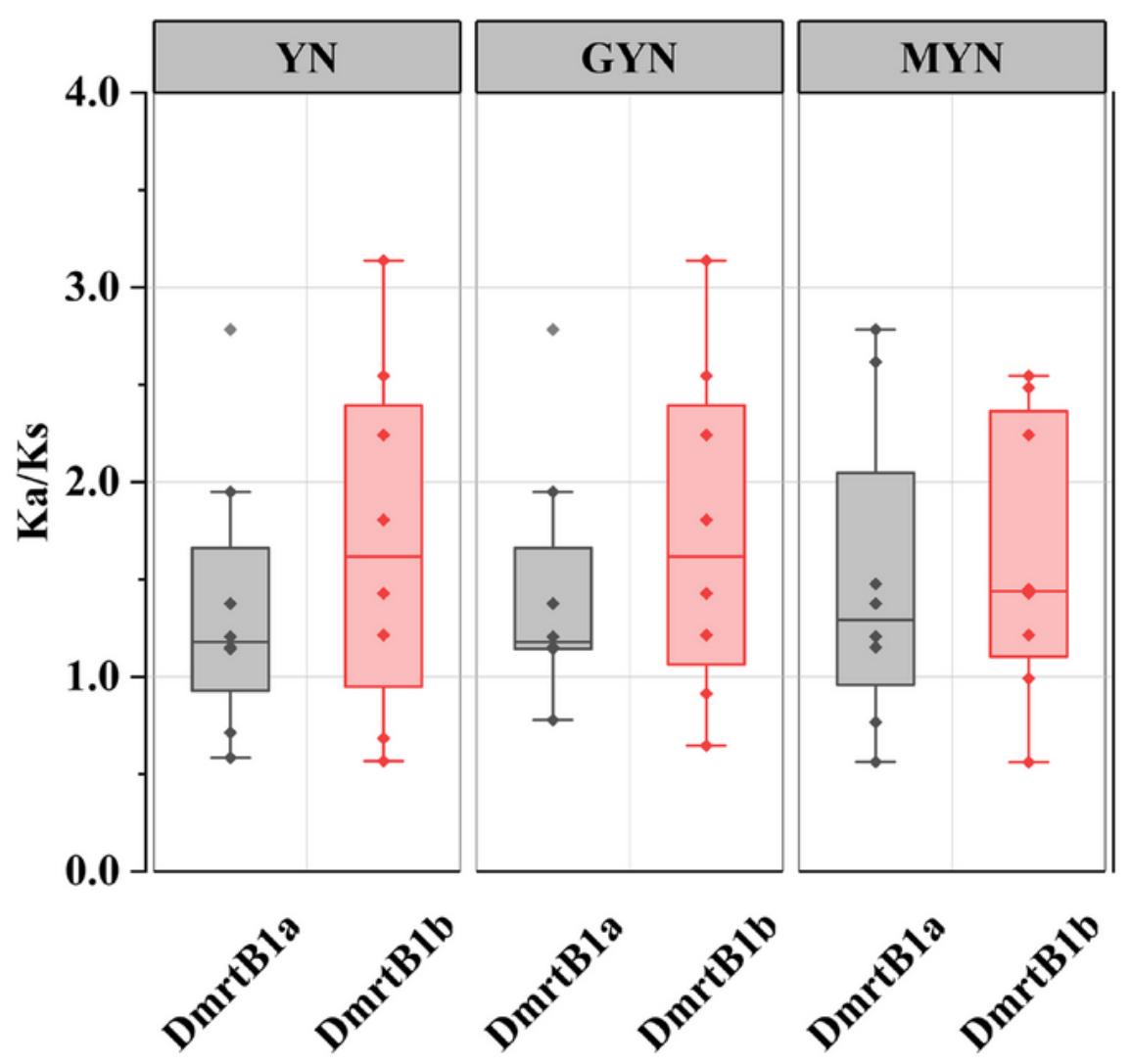

Figure 5

Phylogenetic tree construction and evolutionary pressure analysis for DmrtB1. A) The protein-based phylogenetic tree of DmrtB1 genes was reconstructed based on all nine teleost fishes with reported sequences, including six actinopterygian fishes and three sarcopterygian fishes, using the mouse Dmrt8 amino acid sequence as the outgroup. B) Boxplot showing Ka/Ks ratios of DmrtB1 genes assessed at the codon-based nucleotide sequence level. The open reading frame (ORF) sequences of eight teleost fish 
species (the accession numbers in Supplementary Table 2) were aligned against the reference sequence of A. schrenckii.

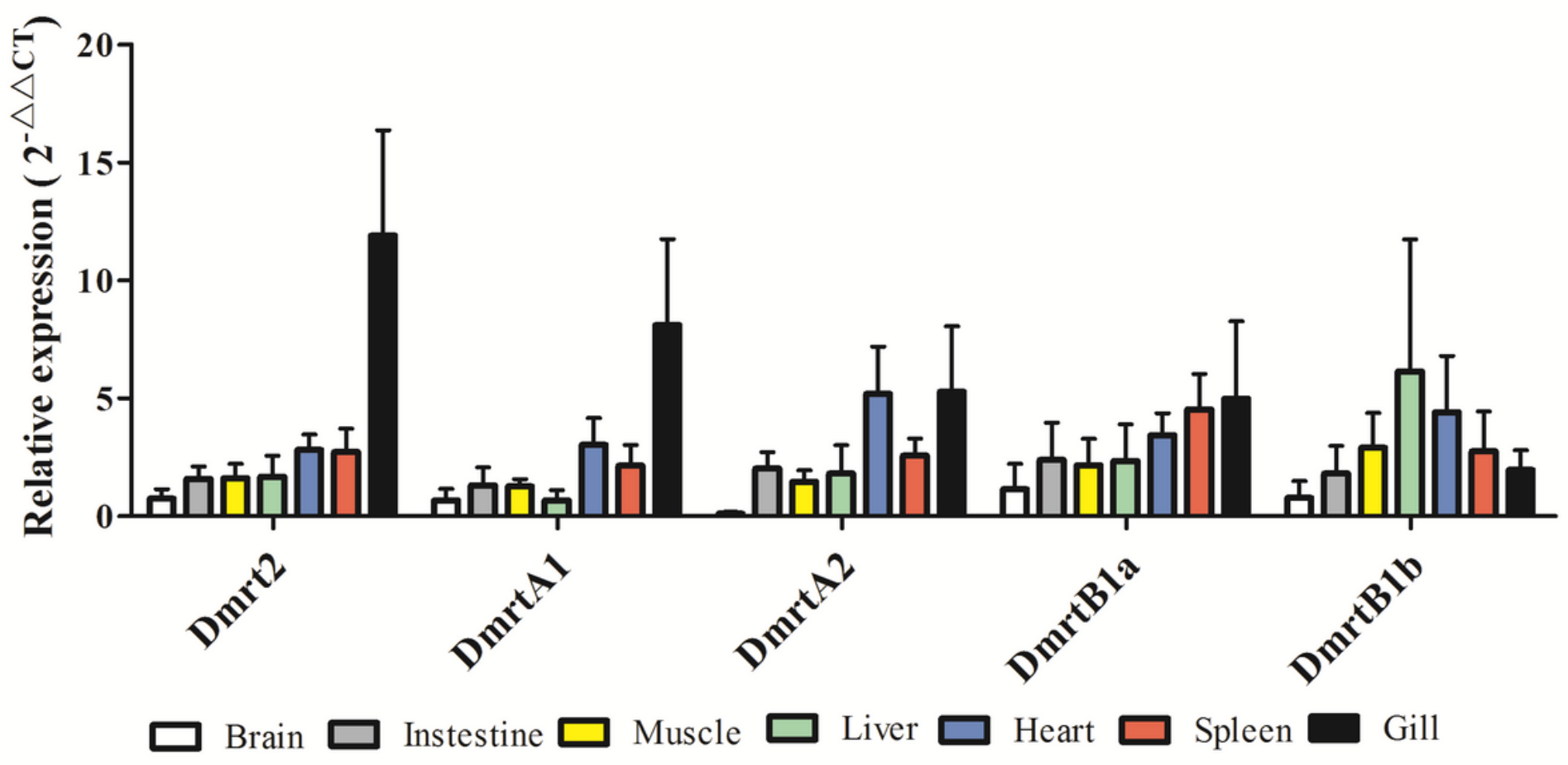

Figure 6

Tissue expression profiling of the five Dmrt family genes in seven main tissues of three-year-old A. schrenckii individuals. 

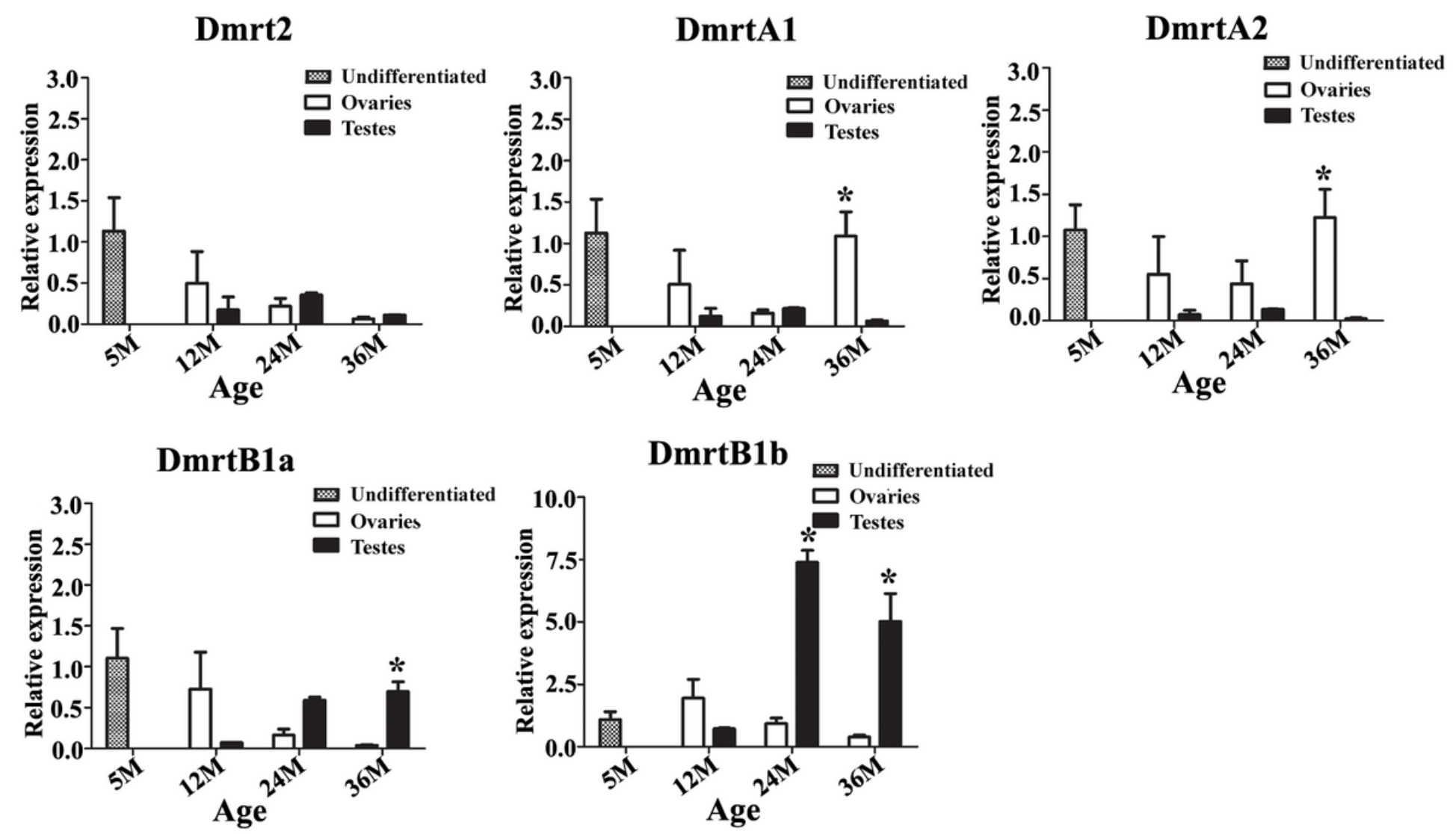

Figure 7

Spatiotemporal expression patterns in the gonads during gonadal differentiation in A. schrenckii. Asterisks $\left({ }^{*}\right)$ above a column represent significant differences between the testis and ovary at the same developmental stage. $P<0.05$ as evaluated by the t-test in SPSS 17.0.

\section{Supplementary Files}

This is a list of supplementary files associated with this preprint. Click to download.

- Supplementaryfiles.rar 